\title{
To Virtualize or Not? The Importance of Physical and Virtual Components in Augmented Reality Board Games
}

\author{
Jessica Ip and Jeremy Cooperstock \\ Centre for Intelligent Machines, \\ McGill University, \\ Montreal, Canada \\ \{Jessica.Ip,Jer\}@cim.mcgill.ca
}

\begin{abstract}
Whereas traditional games have employed entirely physical interfaces, computer games provide a generally virtual alternative. Motivated by interest in supporting conventional gameplay in the computer environment, we investigate the benefits of game interaction techniques based on gestures applied to tangible objects, comparing the user experience with that resulting from more virtualized interaction. Our study is applied in the context of a German-style board game, Settlers of Catan. Significant findings indicate a preference for tangible components for complex interaction tasks.
\end{abstract}

Keywords: Augmented Reality, Tangible User Interfaces, Augmented Gaming, Multi-user Interaction.

\section{Introduction}

Computer games can be extremely powerful and dynamic, simulating complex physical environments, modeling situations that are impossible in real-life, and taking on the role of either moderator, intelligent opponent, or both. Moreover, the computer can automate repetitive, mundane tasks such as shuffling, point-counting, and memorizing rules, factors that may detract from the enjoyment of certain game genres such as board games. Due to these capabilities, we are interested to see if the gaming experience of social board games may be improved with computer augmentation. We hope to discover new game design techniques that will appeal to wider audiences.

\section{Related Work}

As an alternative to both traditional board games and purely computer-focused video games, hybrid board/video games offer the potential to enhance the social gaming experience of the physical world with the benefits of computer augmentation. This is typically achieved by a tangible user interface (TUI) paradigm, which couples physical game pieces to the computer through some sensing mechanism. Using these pieces as input devices that afford manipulation, spatial reasoning skills can be exercised while parallel operation and collaboration is supported between single and multiple users (Fitzmaurice, Ishii, \& Buxton, 1995). In addition, augmented or mixed 
reality techniques can provide real world interaction in a more integrated manner than can a pure video game. Typically, graphics are projected into the same space where the tangible interfaces are used, e.g., on a table. The combined interaction-display format of tangible user interfaces with augmented reality, or Tangible Augmented Reality (TAR) (Billinghurst \& Kato, 2002), enhances face-to-face communications of multi-player games, while allowing players to interact in a natural manner. This idea of merging pre-existing, everyday knowledge with digital interaction techniques arises from the Reality-Based Interaction (RBI) framework, which provides techniques for analyzing and comparing components used for this style of interaction (Jacob, et al., 2008).

\section{Experiments}

Within this RBI framework, we designed a TAR system to investigate the important question of whether tangible or digital components are preferred in social gaming situations. In general, we believe tangible components will be favored due to their affordances for natural interaction. Our hypothesis is that non-strategic (or "set") actions such as game setup and piece sorting will favor automation through virtualization. Moreover, for situations where strategy or multi-player interaction is required, we expect that tangible objects will be preferred due to their assistance with planning through spatialization (Kirsh \& Maglio, 1994) (Patten \& Ishii, 2000) and support of parallel actions between multiple people (Fitzmaurice, Ishii, \& Buxton, 1995). To test this hypothesis, we designed a Settlers of Catan TAR prototype that facilitates the use of tangible objects in addition to automating and assisting players on low-cognition tasks. Traditionally, Settlers is a multi-player strategy board game that involves cooperation, negotiation, and logic.

\subsection{Settlers of Catan Prototype}

The comparison study for Settlers of Catan is presented on the classic board game version, our developed TAR version, and a digitized version on the Apple iPad. As we move from the classic to the digitized version, the number of tangible components decreases as the amount of automation and rule enforcement increases. Fewer rules are enforced when tangible components are present to accommodate rich object manipulation techniques. For tasks where no tangible handle is available, computer automation and rule enforcement are used to guide and inform the player of the actions available.

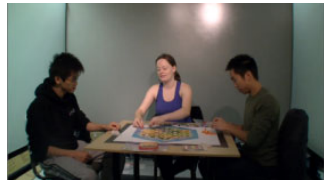

(a)

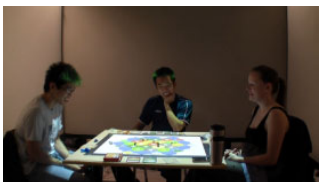

(b)

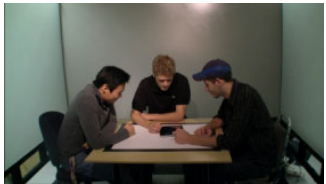

(c)

Fig. 1. Settlers of Catan Classic, TAR, and Digital mode 


\subsection{Design}

The experiment comprised the three conditions as described above. Our subject pool consisted of nine participants, two female and seven male, ranging from 23 to 30 years of age. The participants were randomly assigned to one of three groups of three players. The groups were presented with every condition, with each group experiencing the conditions in a different order. A modified Latin square was used where each condition appeared in every slot of the ordering. The post-test questionnaire was completed immediately after each gameplay session. Once all three conditions were complete, players were asked to choose what they considered to be the best overall condition. The post-test questionnaire was comprised of questions from FUGA's Game Experience and Social Presence in Gaming Questionnaire (GEQ and SPGQ) (de Kort, IJsselsteijn, \& Poels, 2007) (IJsselsteijn, et al., 2008) and O'Brien's User Engagement Scale (O'Brien, 2010).

\section{Results and Analysis}

The questionnaire was comprised of two high level categories: personal game experience and social experience. These reflect the single-user experience and between-player experience, respectively. One or more questions make up each of the listed sections in both personal and social game experience. The responses within each category were averaged to form the associated mean score, as shown in Table 1, separated by subject and condition for analysis. As seen, the TAR condition was rated the highest most frequently in the questionnaire. In general, players found tangible components essential for negotiation and resource trading and preferred automatic, organized board setup.

Table 1. Mean score averaged over all subjects

\begin{tabular}{|l|c|c|c|}
\hline \multicolumn{3}{|c|}{ Questionnaire Results } \\
\cline { 2 - 4 } & \multicolumn{3}{l}{ Mean } \\
\cline { 2 - 4 } & Classic & Digital & TAR \\
\hline Personal Game Experience & 3.04 & 2.11 & 3.59 \\
\hline Perceived Usability & 3.94 & 3.56 & 4.28 \\
\hline Aesthetics & 3.67 & 2.89 & 4.44 \\
\hline Focused Attention & 3.78 & 2.94 & 3.83 \\
\hline Sensory and Imag. Immersion & & & \\
\hline Endurability & 4.33 & 2.83 & 4.50 \\
\hline Negative Affect & 2.78 & 1.78 & 3.00 \\
\hline Positive Affect & 4.17 & 3.28 & 4.50 \\
\hline Social Experience & 3.33 & 3.19 & 3.83 \\
\hline Empathy & 3.37 & 3.22 & 2.96 \\
\hline Negative Feelings & 3.85 & 3.26 & 3.89 \\
\hline Behavioural Involvement & \multicolumn{3}{|l}{} \\
\hline
\end{tabular}




\section{Conclusion}

The study described in this paper tested different implementations of Settlers of Catan to determine the appropriateness of digitizing various physical elements commonly used in board games. Our approach in this regard is to retain tangible components used for making strategic choices while digitizing pieces used for simple, mundane tasks such as the ones required for board setup. These qualities resulted in the TAR condition being the highest rated in many portions of the survey and unanimously chosen by players as their favorite. Preference of tangible components over digital equivalents for complex interaction tasks such as card trading was demonstrated by the significant differences between the virtual and tangible (identical in both classic and TAR) conditions in many areas of the questionnaire.

\section{References}

1. Billinghurst, M., Kato, H.: Collaborative Augmented Reality. Communications of the ACM, 64-70 (2002)

2. de Kort, Y., IJsselsteijn, W., Poels, K.: Digital games as social presence technology: Development of the Social Presence in Gaming Questionnaire, SPGQ (2007)

3. Fitzmaurice, G., Ishii, H., Buxton, W.: Bricks: laying the foundations for graspable user interfaces. In: Proc. SIGCHI Conference on Human Factors In Computing Systems, pp. 442-449 (1995)

4. IJsselsteijn, W., van den Hoogen, W., Klimmt, C., de Kort, Y., Lindley, C., Mathiak, K., et al.: Measuring the experience of digital game enjoyment. In: Proceedings of the 2008 Measuring Behavior Conference (2008)

5. Jacob, R.J., Girouard, A., Hirshfield, L.M., Horn, M.S., Shaer, O., Solovey, E.T., et al.: Reality-based interaction: a framework for post-WIMP interfaces. In: CHI 2008, pp. 201210. ACM, New York (2008)

6. Kirsh, D., Maglio, P.: On distinguishing epistemic from pragmatic action. Cognitive Science, 513-549 (1994)

7. O'Brien, H.L.: Is there a universal instrument for measuring interactive information retrieval?: the case of the user engagement scale. In: IIiX 2010, pp. 335-340. ACM, New York (2010)

8. Patten, J., Ishii, H.: A comparison of spatial organization strategies in graphical and tangible user interfaces. In: Proc. Designing Augmented Reality Environments, pp. 41-50. ACM, New York (2000) 